

\title{
Zur Herstellung von OSB aus originär dauerhaften Holzarten für die Anwendung im frei bewitterten Außenbereich
}

\author{
Sebastian Treml, Fritz Tröger
}

\section{To cite this version:}

Sebastian Treml, Fritz Tröger. Zur Herstellung von OSB aus originär dauerhaften Holzarten für die Anwendung im frei bewitterten Außenbereich. European Journal of Wood and Wood Products, 2010, 69 (1), pp.163-165. 10.1007/s00107-009-0400-7 . hal-00561302

\section{HAL Id: hal-00561302 \\ https://hal.science/hal-00561302}

Submitted on 1 Feb 2011

HAL is a multi-disciplinary open access archive for the deposit and dissemination of scientific research documents, whether they are published or not. The documents may come from teaching and research institutions in France or abroad, or from public or private research centers.
L'archive ouverte pluridisciplinaire HAL, est destinée au dépôt et à la diffusion de documents scientifiques de niveau recherche, publiés ou non, émanant des établissements d'enseignement et de recherche français ou étrangers, des laboratoires publics ou privés. 
EJWWP400_source

Treml, Sebastian; Holzforschung München (WZW)

Tröger, Fritz; Holzforschung München (WZW)

\section{Zur Herstellung von OSB aus originär dauerhaften Holzarten für die Anwendung im frei bewitterten Außenbereich}

\section{Subject}

In an exploratory study, durable wood species were used to manufacture OSB. The chipping quality of these wood species when preparing strands as well as the gluability with phenolic resin was examined.

\section{Material und Methode}

Es stand frisches Rundholz der Holzarten Robinie (Robinia pseudoacacia Linn.), Edelkastanie (Castanea sativa Mill.), Douglasie (Pseudotsuga taxifolia Britt.) und Lärche (Larix europaea Mill.) zur Verfügung. Als Klebstoff wurde das Phenolharz Bakelite PF 2506 HW E der Fa. Hexion Specialty Chemicals TM verwendet.

Das angelieferte frische Rundholz wurde zu 180mm langen und 20/14mm dicken Brettchen aufgetrennt und bis zur Zerspanung nass gelagert. Die Zerspanung erfolgte mit einem Flachscheibenzerspaner Typ Bezner 5481 (Abb. 1), der Messerüberstand wurde zwischen $0,8 \mathrm{~mm}$ und $1,0 \mathrm{~mm}$ variiert. Die Zuführung der Schmalfläche der Brettchen an die Schneiden erfolgte geführt über eine pneumatische Zuführeinrichtung, welche einen konstanten und definierten Anpressdruck an die Scheibe sicherstellt. 


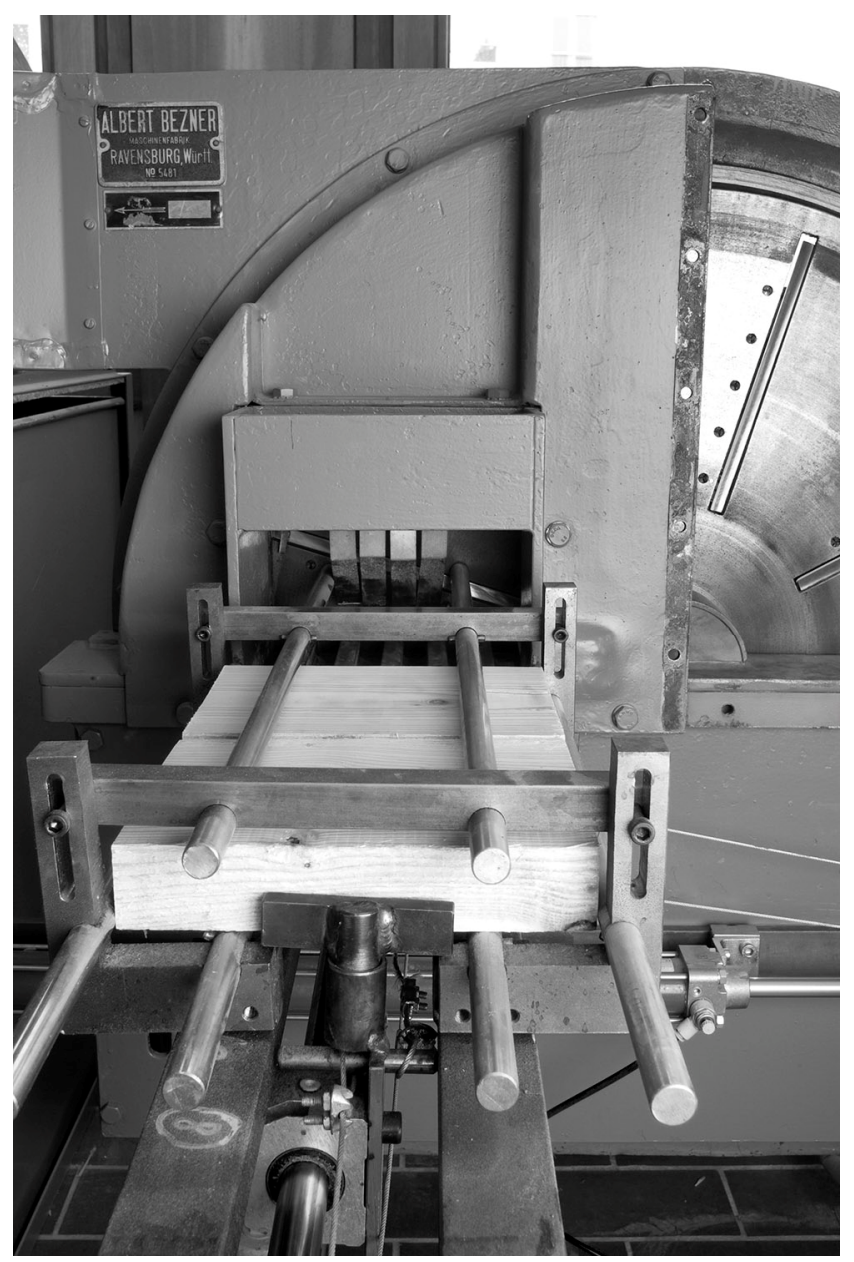

Abbildung 1: Flachscheibenzerspaner mit pneumatisch betriebener Zuführeinrichtung

Figure 1: Flat disc chipper with pneumatically driven feeder

Nach der Zerspanung wurde das Spangut in einem Frischluft- I Ablufttrockner bei $80^{\circ} \mathrm{C}$ auf eine Zielfeuchte von $3 \%$ getrocknet. Die Beleimung erfolgte in einer großvolumigen Beleimtrommel durch eine Zweistoffdüse. Bezogen auf atro Spanmasse wurde für Deck- und Mittelschicht einheitlich ein Feststoffgehalt des Phenolharzes von $10 \%$ angestrebt. Eine Orientierung der Strands während der anschließenden Mattenbildung wurde über eine hierfür entwickelte Einstreuvorrichtung realisiert, die aus einem federnd gelagerten Metallrost besteht, der über einen oszillierenden Druckluftzylinder zu Schwingungen angeregt wird. Über eine kontinuierliche Anpassung der Einstreuhöhe wird ein gleichmäßiger Mattenaufbau und ein einheitlicher Orientierungsgrad der Strands bei den Deckschichten und der Mittelschicht realisiert. Das Verhältnis der Decklagen zur $90^{\circ}$ gedrehten Mittellage beträgt 60:40. Die fertig gestreute Matte wurde anschließend in einer hydraulisch 
betriebenen Heißpresse bei $180^{\circ} \mathrm{C}$ verpresst. Der Presszeitfaktor betrug $20 \mathrm{~s} / \mathrm{mm}$.

\section{Resultate}

Mit allen Holzarten wurden Zerspanungsversuche mit variierenden Zerspanungsparametern durchgeführt. Es zeigte sich, dass die Zerspanung der beiden Nadelhölzer Lärche (LÄ) und Douglasie (DG) problemlos möglich ist. In beiden Fällen konnten geometrisch definierte Strands erzeugt werden, die für die OSB Herstellung geeignet sind. Die beiden Laubhölzer verhielten sich bezüglich der Strandqualität problematisch. Vor allem die Edelkastanie (EKE) neigt hier bei ungünstiger Jahrringlage, d.h. liegenden Jahrringen im zu zerspanenden Rohbrettchen, überwiegend entlang des grobporigen Frühholzbereiches zum Bruch, was eher zu Spreißeln und weniger zu flächigen OSB Strands führt (Abb. 2).
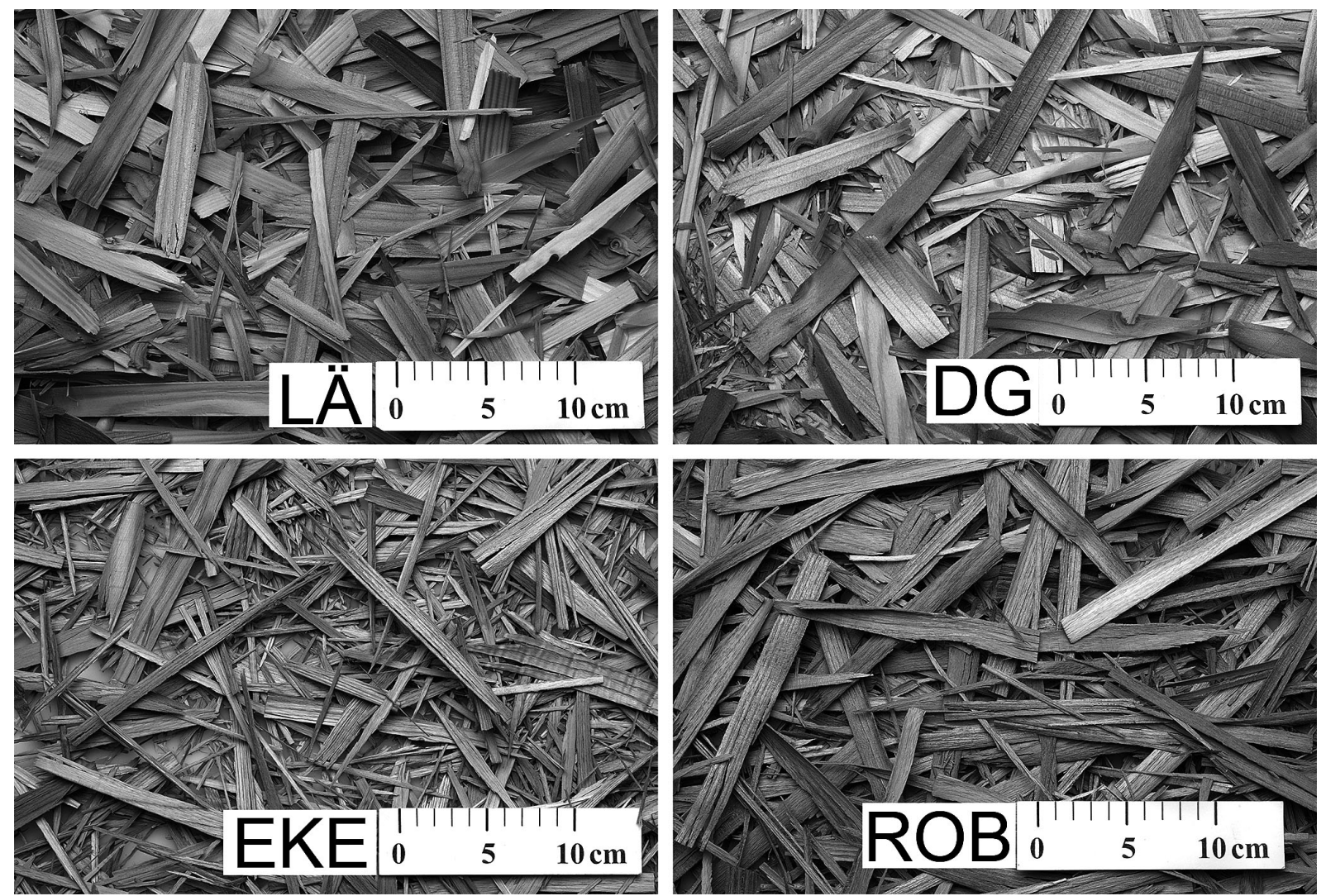

Abbildung 2: Erzeugte Strands unterschiedlicher Holzarten (Sollabmessungen L:180; B:20; D:1 $[\mathrm{mm}])$

Figure 2: Strands prepared from different wood species $(1: 180 ; \mathrm{w}: 20 ; \mathrm{t}: 1(\mathrm{~mm}))$ 
Aus den Holzarten Robinie (ROB) und Edelkastanie wurden schließlich Platten mit den Abmessungen 500mm x 500mm x 19mm/13mm hergestellt. Die Verklebung mit dem verwendeten Phenolharz war unproblematisch. $\mathrm{Zu}$ beachten ist in jedem Fall eine Anpassung der Zielrohdichte der fertigen Platte an die Rohdichte der verwendeten Holzart. Die Rohdichte von Robinie zu Kiefer (als Referenzholzart in der industriellen Anwendung) verhält sich in etwa im Verhältnis 1:1,5. Diesen Faktor auf die Zielrohdichte der Platte zu übertragen ist wahrscheinlich nicht zweckmäßig. Holzartenspezifisch liegt ein unterschiedliches Querdruckverhalten vor (SCHWAB 1986), das nicht zwangsläufig mit der Rohdichte korreliert ist (KOLLMANN 1951), sich aber auch im Zusammenhang mit der Jahrringlage auf das Verdichtungsergebnis und damit weiter das Rohdichteprofil auswirken kann. Zudem führen hohe Rohdichten $\mathrm{zu}$ einem hohen $\mathrm{m}^{2}$-Gewicht der fertigen Platte, was schließlich die Handhabbarkeit und Verarbeitungsfreundlichkeit des fertigen Produktes einschränken kann. Orientierend wurden Platten mit einer Rohdichte zwischen $630 \mathrm{~kg} / \mathrm{m}^{3}$ und $800 \mathrm{~kg} / \mathrm{m}^{3}$ hergestellt. Ab einer Rohdichte, die um ca. $10 \%$ über der Rohdichte der eingesetzten Holzart lag, konnte in den Deckschichten ein ausreichend geschlossener Spanverbund erzielt werden.

\section{Ausblick}

Bezüglich der Festlegung eines möglichen und sinnvollen Verdichtungsverhältnisses in Abhängigkeit von der Rohdichte und des Querdruckverhaltens der eingesetzten Holzart besteht weiterer Untersuchungsbedarf. In diesem Zusammenhang sollen auch Plattenvarianten hergestellt werden, die durch die Verwendung von unterschiedlicher Rohdichte in Deck- und Mittelschicht eine ausgeprägte Verdichtung in den Deckschichten aufweisen. Die hergestellten Platten sollen im Vergleich mit industriell und weiterem labormäßig hergestelltem Referenzmaterial einer Freibewitterung ausgesetzt werden, um den 
EJWWP400_source

Einfluss der Holzart auf die Dauerhaftigkeit daraus hergestellter OSB für den Außenbereich nachzuweisen.

\section{Literatur}

KollmanN, F, 1951: Technologie des Holzes., Berlin, Springer

SCHWAB, E, 1986: Kennwerte des Querdruckverhaltens von Laubhölzern.

Holz Roh- Werkst 44, 259-269 